\title{
INFECCIÓN DE Genypterus blacodes (SCHNEIDER, 1801) POR EL COPÉPODO PARÁSITO Sphyrion laevigatum (QUOY \& GAIMARD, 1824)
}

\section{INFECTION BY Genypterus blacodes (SCHNEIDER, 1801) BY PARASITIC COPEPOD Sphyrion laevigatum (QUOY \& GAIMARD, 1824)}

Paulina Donoso.
$\begin{aligned} & \text { Laboratorio ADL Diagnostic Chile Ltda, sede Villarrica, email: } \\ & \text { paulinadonoso.diaz@gmail.com }\end{aligned}$

Recibido: 03 de febrero de 2017; Aceptado: 07 de abril de 2017.

\section{RESUMEN}

Con el propósito de contribuir a la ecología de Sphyrion laevigatum (Quoy \& Gaimard, 1824) en Genypterus blacodes (Schneider, 1801) se estudió la posible existencia de un patrón espacial, pudiendo ser aleatorio, uniforme o agregado para este copépodo parásito. La investigación se realizó en Supermercado Jumbo, Feria Pinto y Mercado Central, Temuco, IX Región, Chile ( $\left.38^{\circ} 44^{\prime} 24^{\prime \prime} \mathrm{S}, 72^{\circ} 35^{\prime} 24^{\prime \prime} \mathrm{W}\right)$ durante los meses de Junio y julio del 2012. Para determinar la distribución del copépodo parásito S. laevigatum se procedió a observar la superficie corporal de 209 G. blacodes estableciendo la cantidad de parásitos por pez, y con estos datos se obtuvo la relación varianza / media. Los resultados mostraron presencia del parásito en la zona, sin embargo, ésta fue baja en el período de estudio, alcanzando solo un $18 \%$ de la muestra total positiva a $S$. laevigatum. El número mínimo de este copépodo parásito encontrado en $G$. blacodes fue de 1 y el máximo de 2.

Palabras clave: Genypterus blacodes, Sphyrion laevigatum, ectoparásitos. 
Sustainability, Agri, Food and Environmental Research, 5(1), 2017: 33-42

ISSN: 0719-3726

\section{ABSTRACT}

In order to contribute to the ecology of Sphyrion laevigatum (Quoy \& Gaimard, 1824) in Genypterus blacodes (Schneider, 1801) the possible existence of a spatial pattern was studied, being it possible to be random, uniform or aggregate for this parasite copepod. The research was carried out at the Jumbo Supermarket, Pinto Fair and Mercado Central, Temuco, IX Region, Chile (38 ${ }^{\circ} 44^{\prime} 24$ "S, $72 \circ 35^{\prime} 24^{\prime \prime}$ W) during the months of June and July of 2012. To determine the distribution of the parasite copepod S. laevigatum was observed to the body surface of 209 G. blacodes establishing the amount of parasites per fish, and with these data the variance / average relation was obtained. The results showed presence of the parasite in the area, but this was low in the study period, reaching only $18 \%$ of the total positive sample to S. laevigatum. The minimum number of this parasite copepod found in $\mathrm{G}$. blacodes was 1 and the maximum number was 2 .

Key words: Genypterus blacodes, Sphyrion laevigatum, ectoparasites

\section{INTRODUCCIÓN}

La especie G. blacodes (Schneider, 1801), conocida también como "congrio dorado", pertenece a la familia Ophidiidae del orden de Ophidiiformes y es un pez demersal bentónico con distribución en la costas de Chile, Argentina y Nueva Zelandia (Bahamonde y Zavala, 1981; Grabda y Ślósarczyk, 1981; Garciarena, 1989; Chong y Aguayo, 1990; Díaz de Astarloa y Figueroa, 1993; Horn, 1993; Cordo, 2001; Horn, 2005; Torres et al., 2006). En Chile está presente desde la región de Coquimbo a $30^{\circ} \mathrm{S}$ hasta el extremo sur del país a $57^{\circ} \mathrm{S}$ (Ojeda et al., 2000; Subpesca, 2011), entre los 50 y 500 metros de profundidad (Bahamonde y Zavala, 1981; Renzi, 1986).

Se han realizado investigaciones de diversa índole sobre $G$. blacodes (Schneider, 1801), entre ellos se destaca su fauna parasitaria, aspectos de su biología, fecundidad, madurez sexual, crecimiento, dieta, estructura y distribución de su población (Grabda y Ślósarczyk, 1981; Bahamonde y Zavala, 1981; Renzi, 1986; Garciarena,1989; Chong y Aguayo, 1990; Ho,1992; Sardella et al, 1998; Brickle et al, 2003; Horn, 2005; Torres et al, 2006; Wiff et al., 2007; Freijo, et al., 2009; Gonzales et al., 2009; Canales et al, 2010; Wiff et al, 2011). Sin embargo, no se tiene registro de investigaciones que consideren los parámetros ecológicos de los ectoparásitos presentes en el congrio dorado perteneciente a la unidad pesquera del sur de chile, aspecto que se aborda en las siguientes páginas de este escrito. 
La parasitosis constituye una pérdida económica significativa, ya sea, por la prevención, disminución de rentabilidad de la explotación por mortalidad o por descensos en los rendimientos, y reducción de la calidad que se exige para la exportación a la población mundial (Kinkelin et al, 1991).

Los parásitos causan diversos daños en el organismo del hospedador, afectando a las branquias, piel, músculos, esqueleto, órganos sensoriales y órganos internos (Kabata, 1970), incluso son capaces de producir enfermedades agudas o la muerte, dependiendo del número de parásitos, especie, tamaño y estado de salud del hospedador (Needham y Wootten ,1981). Con respecto a los copépodos y las sanguijuelas, éstos poseen la capacidad de implantarse más profundamente que otros ectoparásitos provocando ruptura tegumentaria, hemorragias y necrosis subcutáneas, las cuales son relativamente compensadas por las reacciones reparadoras del hospedador. Además, los antígenos salivales y fecales producidos por ectoparásitos pueden estimular respuestas inmunes en algunos hospedadores y actuar como vectores para el desarrollo de infecciones secundarias (Kinkelin, et al, 1991).

Los ejemplares de G. blacodes (Schneider, 1801), son parasitados por ectoparásitos copépodos de la especie Sphyrion laevigatum (Quoy \& Gaimard, 1824) (Grabda y Ślósarczyk, 1981; Ho, 1992; Sardella et al., 1998; Brickle et al, 2003). La distribución de S. laevigatum (Quoy y Gaimard, 1824) se limita a los océanos que se encuentran al sur del paralelo 30S, comprendiendo los mares de Argentina, Nueva Zelandia, Chile, África y Australia (Ho, 1992; Grabda y Ślósarczyk, 1981).

\section{MATERIALES Y METODOS}

El presente estudio se realizó en el "Mercado Central, Supermercado Jumbo y Feria Pinto" (38 $\left.44^{\prime} 24^{\prime \prime S}, 72^{\circ} 35^{\prime} 24^{\prime \prime} \mathrm{W}\right)$, ubicado en la provincia de Cautín, ciudad Temuco, IX Región de la Araucanía - Chile, durante los meses de Junio y Julio del año 2012. Se examinó la piel, aletas y cámara branquial de 209 ejemplares de congrios dorados (G. blacodes) que se comercializaban en la ciudad. Posteriormente de realizar el conteo de los copépodos parásitos encontrados sobre la musculatura de los peces, los parásitos se almacenaron en un frasco con alcohol 70\% para su preservación (Sardella, et al., 1998; Turner et al, 2003; Castro y González, 2009).

Los resultados obtenidos fueron analizados con técnicas de estadística descriptiva la cual se utilizó para describir el patrón de distribución de los parásitos en los G. blacodes. Se utilizó la relación varianza/ media de la población para caracterizar 
Sustainability, Agri, Food and Environmental Research, 5(1), 2017: 33-42

ISSN: 0719-3726

los datos como patrón al azar, uniforme o agrupado. Posteriormente se ajustaron los resultados al modelo probabilístico más acorde, con el objetivo de establecer si los datos se adaptan a la prueba de distribución binomial positiva, distribución de Poisson o distribución binomial negativa (Zar, 1999; Fernandes et al., 2003).

\section{RESULTADOS}

De los 209 ejemplares de G. blacodes que se examinaron, solo 38 peces estaban parasitados y 171 peces se encuentran libres de S. laevigatum. Por lo tanto, del total de los peces, el $18 \%$ se encuentra positivos a la infestación por el parásito copépodo, resultando $82 \%$ de G. blacodes negativos a la presencia de S. laevigatum. Con respecto, al número de copépodos parásitos encontrados por pez, se establece que 29 peces presentaron 1 parásito, 9 peces poseían 2 parásitos y en 171 ejemplares se observaron 0 parásito en la superficie de su cuerpo, recolectándose un total de 47 especímenes de S.laevigatum.

El cálculo del índice de dispersión, donde la varianza $(=0,261685683$ y la media ( $m=0,224880383$ ) siendo el valor del índice de dispersión de 1,163666 con lo cual se propone la distribución agrupada para S. laevigatum.

\section{DISCUSIÓN}

El grado de infección de Shyrion laevigatum en ejemplares de G. blacodes alcanza el $18 \%$ del total de la muestra, variando este resultado con la investigación realizada en las Islas Malvinas (Brickle et al, 2003), en la cual se establece que aproximadamente un $48 \%$ de la muestra se ve afectada por estos parásitos, pudiéndose atribuir a la mayor prevalencia de la fauna parasitaria de G. blacodes de Argentina, localización geográfica y cantidad del copépodo en el medio acuático (Sardella et al, 1998). Además, se ha establecido que la presencia de una especie parásita en el hospedador está determinada por la susceptibilidad del hospedador, edad, sexo, respuesta inmunitaria, resistencia y tamaño del huésped (Needham y Wootten, 1981; Jackson, et al., 2006; Sardella et al, 1998), como por las características físicas y bióticas del medio ambiente y distribución batimétrica de los peces.

Con respecto a la cantidad de S. laevigatum presentes en G. blacodes, ésta concuerda con la descrita por Brickle et al, (2003) en los congrios dorado provenientes 
de las islas Malvinas, en cuyo estudio se estableció que la mayoría de los peces infectados presentaron entre 1 y 2 parásitos, sin embargo discrepan en el máximo de parásitos observados, ya que un 2,2 \% de peces poseía más de 5 parásitos en comparación con este estudio en que el máximo fue de 2 parásitos. Otros estudios realizados en Sudáfrica, han establecido que el máximo de Sphyrion laevigatum en su forma adulta en Genypterus capensis fue de 8 parásitos. Brickle et al, (2003), menciona que estas diferencias se deben a las diversas características geográficas y cantidad del copépodo en el medio acuático.

Si bien, otros estudios realizados sobre la presencia de parásitos en $G$. blacodes, han establecido que existe relación entre el tamaño de los peces y el número de parásitos en ellos, Brickle, et al, (2003) determinaron que el tamaño no influye en la cantidad de parásitos por pez y que la edad tendría un papel más relevante al correlacionarla con la abundancia media.

Sardella, et al, (1998) declaró una correlación significativa entre la longitud del anfitrión y la prevalencia y la media de intensidad de $S$. laevigatum. Además, se estableció que existieron diferencias de tamaños entre la zona norte y sur de Argentina. Éstas discrepancias de tamaño y número de parásitos en el hospedador, puede deberse a la distribución batimétrica y geográfica de G. blacodes.

El hospedador frente a la presencia de parásitos, genera reacciones inmunes, causando además cambios en el comportamiento, disminución de su capacidad de defensa frente a depredadores, reducción de su eficiencia biológica (fecundidad y crecimiento) y capacidad competitiva del hospedador (Kinkelin, et al, 1991; Begon et al, 1999). La cantidad de parásitos encontrados en G. blacodes en este estudio es reducido, existiendo un bajo riesgo de generar en estos peces reacciones graves y /o mortales, sin embargo, pudieron haber provocado pequeños cambios en el comportamiento, como por ejemplo, mayor cantidad de tiempo empleado en el frotamiento y menor tiempo en alimentarse.

El cefalotórax de Sphrion ssp. está anclado profundamente en la musculatura de su hospedero, y la reacción limitada de esta frente a la infestación produce la formación de un quistes color marrón - gris. Las lesiones que produce Sphrion ssp comúnmente son estéticas, ya que el cefalotórax de los parásitos muertos permanecen en la musculatura y son encapsulados por los tejidos del hospedador, produciendo obsesos de hasta $2 \mathrm{~cm}$ de largo.

La distribución agregada indica que muchos peces presentan a pocos parásitos o a ningún individuo parásito, mientras que pocos hospedadores presentan un gran número de éstos (Begon, et al, 1999; Boada, et al., 2012; Poulin, 2007). Se ha 
Sustainability, Agri, Food and Environmental Research, 5(1), 2017: 33-42

ISSN: 0719-3726

indicado que esta distribución es característica de los procesos parasitarios (Iannacone, et al., 2000; Iannacone y Alvariño,2009; Iannacone, et al, 2010; Iannacone, et al., 2011; Iannacone, et al., 2012; Boada, et al., 2012; Luque, 1994), y puede estar causada por diversas razones, tales como : a) multiplicación de parásitos en los hospederos infectados al azar; b) dispersión baja o lenta produciendo agregaciones de parásitos localmente densas; c) variabilidad en la susceptibilidad de los hospederos a la infección, a causa de factores genéticos, ambientes o de comportamiento (Begon, et al, 1999)

Según Anderson y May (1978) los parásitos que presentan una distribución agregada en el hospedador están relacionados con diversos factores. Los principales factores que se podrían atribuir a los diversos patrones de distribución son el estado inmunitario y la heterogeneidad de la población hospedera. También, menciona que la población de parásitos sobre el hospedador puede mantenerse por la relación mortalidad/natalidad de los parásitos condicionada por la inmunidad que desarrolla el hospedador para su defensa y la competencia intraespecífica de los parásitos por el espacio y nutrientes en el huésped.

Se considera que los parásitos pueden tener un papel importante al proporcionar equilibrio e influir en la forma y la organización de las comunidades (Anderson y May, 1978), ya que regulan la abundancia y/o densidades de las poblaciones hospederas (Anderson y May, 1978), sin embargo, pueden amenazar a las especies en peligro de extinción (Hudson et al, 2006). Además, estos parásitos desempeñar la función de estabilizadores de la red alimentaria y son indicadores de un ecosistema saludable (Hudson et al, 2006; Morales-Serna y Gómez, 2012).

\section{REFERENCES}

Anderson, R y May, R., 1978. Regulation and stability of host-parasite population interactions. Journal of Animal Ecology, 47: 219-247.

Bahamonde, N y Zavala, P., 1981. Contenidos gástricos de Genypterus maculatus (Tschudi) y Genypterus blacodes (Schneider) capturados en Chile entre $31^{\circ} \mathrm{y}$ $37^{\circ} \mathrm{S}$ (Teleostomi, Ophidiidae), Boletín del Museo Nacional de Historia Natural, Chile, 38: 53-59.

Begon, M., Harper, J. y Townsend, C., 1999. Ecología: individuos, poblaciones y comunidades. $3^{a}$ ed. Omega, Barcelona, España. 1148 pp. 
Boada, M., Bashirullah, A., Marcano, J., Alió, J.y Vizcaíno, G., 2012. Estructura comunitaria de ectoparásitos en branquias del jurel Caranxhippos (Linnaeus, 1776) en Santa Cruz y Carúpano, Estado Sugre, Venezuela, Revista Científica, FCV-LUZ, 22: 259-272.

Brickle, P., Buxton, N y Villalón, E., 2003. Infection of Sphyrion laevigatum (Copepoda: Sphyriidae) on Genypterus blacodes (Pisces: Ophidiidae) from the Falkland Islands, South Atlantic, The Journal of Parasitology, 89: 242-244.

Canales, C., Ferrada,S., Hernández,C y Galleguillos, R., 2010. Population structure and demographic history of Genypterus blacodes using microsatellite loci. Fisheries Research, 106: 102-106.

Castro, R y Gonzalez, M., 2009. Two new species of Clavella (Copepoda, Siphonostomatoida, Lernaeopodidae) and new species of Lophoura (Copepoda, Siphonostomatoida , Sphyriidae): parasites on the deep-water fish, Nezumiapulchella fron the northern chilean coast. Crustaceana, 82: 411-423.

Chong, J y Aguayo, M., 1990. Determinación de edad y estimación de los parámetros del crecimiento del congrio dorado, Genypterus blacodes (Schneider, 1801) (Osteichthyes, Ophidiidae) en el Pacífico Sur Oriental, Biología Pesquera, 19: 5567.

Cordo, H, 2001. Estandarización del esfuerzo de pesca ejercido sobre abadejo (Genypterus blacodes), periodo 1986-1996. Revista de Investigación y Desarrollo Pesquero, 14: 79-93.

Diaz De Astarloa, J y Figueroa, D., 1993. Las especies del género Genypterus (Pisces, Ophidiiformes) presentes en aguas argentinas. En:Universidad Católica del Norte. Facultad de Ciencias del Mar, IV COLACMAR. Coquimbo,Chile, 30 Sep-04 Oct 1991. Coquimbo: Chile.

Fernandes, M. Busoli, A y Barbosa, J., 2003. Distribuição Espacial de Alabama argillacea (Hübner) (Lepidoptera:Noctuidae) em Algodoeiro, Neotropical Entomology, 32: 107-115.

Freijo, R., García, A., Portiansky, E., Barbeiti, C., Macchi,G y Díaz,A.,2009. Morphological and histochemical characteristics of the epithelium of ovarian lamellae of Genypterus blacodes (Schneider, 1801). Fish Physiology and Biochemistry, 35: 359-367.

Garciarena, A., 1989. Caracteres distintivos de las especies del género Genypterus (Pisces, Ophidiidae) presentes en aguas argentinas, Seminario de Licenciatura, Universidad Nacional, Facultad de Ciencias Exactas y Naturales, Mar del Plata, p 26. 
Sustainability, Agri, Food and Environmental Research, 5(1), 2017: 33-42

ISSN: 0719-3726

Grabda, J y Ślósarczyk, W., 1981. Parasites of marine fishes fron New Zealand. Acta Ichthyologica et Piscatoria, 11: 85-103.

Gonzáles, E., Aránguiz, A., Ramos, R y Rojas A., 2009. Demographical analysis of the pink ling Genypterus blacodes (Schneider 1801) in the austral demersal fishery: A matrix approach evaluating harvest andnon-harvest states, Fisheries Research, 96: $216-222$.

George-Nascimento, M., Lobos, V., Torrijos, C y Klan, R., 2004. Species composition of assemblages of Ceratomya (Myxozoa), Parasites of lings Genypterus (Ophidiidae) in the southeastern pacific ocean: an ecomorphometric approach. The Journal of Parasitology, 90: 1352-1355.

Ho, J., 1992. Does Sphyrion lumpi (Kryoer) (Copepoda, Sphyriidae) occur in the Sea of Japan ? With Discussion on the origin and Dispersal of Sphyrion Cuvier, 1830), Report of the Sado Marine Biological Station, 22: 37-48.

Hendrix, Ch., 1998. Diagnostic Veterinary Parasitology. 2nd ed. St. Louis: Mosby.

Horn, P., 1993. Growth, age structure, and productivity of ling, Genypterus blacodes (Ophidiidae), in New Zealand waters. New Zealand Journal of Marine and Freshwater Research, 27: 385-397.

Horn, P., 2005.A review of the stock structure of ling (Genypterus blacodes) in New Zealand waters. New Zealand Fisheries Assessment Report, 59, p. 41

Hudson, P., Dobson, A y Lafferty, K., 2006. Is a healthy ecosystem one that is rich in parasites?, TRENDS in Ecology and Evolution, 21: 381-385.

Iannacone, J., Mejía, W., Alcócer, F., Briones, G y Román, A., 2000. Características de la infestación Diplectanumsp. (Monogenea: Monopisthocotylea: Diplectanidae) en el ayanque Cynoscionanalis Jenyns (Pisces: Teleostei: Sciaenidae). Revista Peruana de Biología, 6: 44-54.

Iannacone, J y Alvariño, L., 2009. Dinámica poblacional de la diversidad parasitaria de la "Cabrilla" Paralabrax humeralis (Teleostei: Serranidae) en Chorrillos, Lima, Perú, Neotropical Helminthology, 3: 73-88.

Iannacone, J., Morón, L. y Guizado, S., 2010. Variación entre años de la fauna de parásitos metazoos de Sciaena deliciosa (Tschudi, 1846) (Perciformes: Sciaenidae) en Lima, Perú, Latin American Journal of Aquatic Research, 38: 218226.

Iannacone, J., Avila, J., Rojas, S., Salas, M., Neira, K., Palomares, R., Valdivia, S., Pacheco, A., Benvenutto, V. y Ferrario, V., 2011. Dinámica poblacional de los parásitos metazoos del pez guitarra del pacífico Rhinobatos planiceps (Batoidea: 
Sustainability, Agri, Food and Environmental Research, 5(1), 2017: 33-42

ISSN: 0719-3726

Rajiformes) de la zona costera marina de Lima, Perú. Neotropical Helminthology, 5: 265-278.

Iannacone, J., Sánchez, V., Olazábal, N., Salvador, C., Alvariño, L y Molano, J., 2012. Ecological indices of parasites of Scartichthysgigas (Steindachner, 1876) (Perciformes: Blenniidae) of the coasts of Lima, Peru. Neotropical Helminthology, 6: 191-203.

Jackson, J., Pleass, R., Cable, J., Bradley, J. y Tinsley, R., 2006. Heterogenous interspecific interactions in a host-parasite system. International Journal for Parasitology, 36: 1341-1349.

Kabata, Z., 1970. Diseases of Fishes. Crustacea as enemies of fishes. T.F.H. Publications, Jersey, New Jersey. $171 \mathrm{pp}$.

Kinkelin, P. Michel, C y Ghittino, P., 1991. Tratado de las Enfermedades de los Peces. Acribia, Zaragoza, España. 370 pp.

Luque, J., 1994. Dinámica poblacional y estructura de la comunidad de metazoarios parásitos de Menticirrhus ophicephalus (Pisces: Sciaenidae) en la costa peruana, Revista de Biología Tropical, 42(1/2), pp. 21-29.

Morales-Serna, F. y Gómez, S., 2012. Generalidades de los copépodos parásitos de peces en aguas profundas y el caso de Lophoura brevicollum (Siphonostomatoida: Sphyriidae). En: Zamorano, P., Hendrickx, M y Caso, M (Eds.). Biodiversidad y comunidades del talud continental del Pacífico mexicano. Instituto Nacional de Ecología, Secretaría del Medio Ambiente y Recursos Naturales, pp. 145-158.

Needham, T y Wootten, R., 1981. Parasitología de los teleósteos. En: Roberts, R,1 ed. 1981. Patología de los Peces. Madrid: Mundi- Prensa.

Ojeda, P. Labra, F y Muñoz, A., 2000. Biogeographic patterns of Chilean littoral fishes, Revista Chilena de Historia Natural,73: 625-641.

Poulin, R. 2007. Evolutionary Ecology of Parasites. Princeton University Press. Princeton, Estados Unidos. 214 pp.

Renzi, M., 1986. Aspectos Biológico-Pesqueros del Abadejo (Genypterus blacodes), Revista de Investigación y Desarrollo Pesquero, 6: 5-19.

Sardella, N., Avendaño, M. y Timi, J., 1998. Parasite communities if Genypterus blacodes and G. brasiliensis (Pisces: Ophidiidae) from Argentina, Helminthologia, 35: 209-218.

SUBPESCA (Subsecretaria de Pesca), 2011. Cuota global anual de captura de congrio dorado (Genypterus blacodes), año 2012. [Online] Disponible en: 
http://www.subpesca.cl/institucional/602/articles-79902_documento.pdf (Visited 04th Oct2016).

Torres, E., Manero, A y Vargas, F., 2006. Listado sistemático y distribución temporal de los peces del estuario del rio gallegos (Santa Cruz, Argentina). Anales del Instituto de la Patagonia, 34: 59-64.

Turner, T., Kyne, P y Bennett, M., 2003. Description of Paeonasymbolin. sp. (Copepoda: Sphyriidae), parasitic on Asymbolus spp. (catsharks) and a new host record for $P$. australis Kabata, 1993. Systematic Parasitology, 56: 235-239.

Wiff, R., Ojeda, V y Quiroz, J., 2007. Age and growth in pink cusk-eel (Genypterus blacodes) off the Chilean australzone: evaluating differences between management fishing zones. Journal of Applied Ichthyology, 23: 270-272.

Wiff, R., Quiroz, J., Ojeda, V y Barrientos, M., 2011. Estimación de mortalidad natural e incertidumbre para congrio dorado (Genypterus blacodes Schneider, 1801) en la zona sur-austral de Chile. Latin American Journal of Aquatic Research, 39: 316-326.

Zar, J., 1999. Biostatistical analysis. 4 th ed. Prentice Hall. Englewood Cliffs, New Jersey. 663 pp. 